Reiner Keller

\title{
Kultur als Diskursfeld
}

\section{Deutungsmuster der Abfallpolitik im deutsch-französischen Vergleich}

Der nachfolgende Beitrag stellt theoretische Grundlagen, Vorgehensweisen und Ergebnisse einer vergleichenden Untersuchung öffentlicher Debatten zur Abfallpolitik in Deutschland und Frankreich in den Jahren 1970 - 1995 vor (Keller 1998). Beide Länder galten - und gelten zum Teil weiterhin - als exemplarische Antipoden der Umweltdiskussion: auf der einen Seite die Bundesrepublik Deutschland als umweltpolitisch aufgeregte Vorreiter-Nation, auf der anderen Seite Frankreich als Land mit unerschütterlichem Fortschrittsglauben. Als weithin unverfügbare Grundlage dieser Unterschiede werden oft neben spezifischen demo- und geographischen Gegebenheiten tief verwurzelte kollektive Traditionen - das romantische Naturempfinden hier, die rationalistischen Weltbezüge da - benannt. Demgegenüber wurde in der nachfolgend erläuterten Studie eine streng empirische‘ Haltung eingenommen: $\mathrm{Zu}$ nächst ging es um die unvoreingenommene Rekonstruktion der Inhalte, Strukturen und Verläufe umweltpolitischer Auseinandersetzungen am Beispiel des Problemfeldes ,Umgang mit Hausmüll'. Dabei traten sowohl Ähnlichkeiten wie auch Unterschiede der entsprechenden Diskussionen zu Tage. Auch konnte gezeigt werden, wie unterschiedliche nationale Kulturen des Umweltverhältnisses bzw. der Umweltpolitik sich nicht einfach aus der Tradition heraus ergeben, sondern als Ergebnisse eines permanenten diskursiven (Re-)Produktionsprozesses in den beteiligten institutionellen Feldern begriffen werden können. Nachfolgend möchte ich zunächst die der Untersuchung zugrunde liegenden theoretisch-begrifflichen Überlegungen - insbesondere das Verständnis von Kultur, Diskurs und Deutungsmustern - erläutern. Daran anschließend werden einige Ergebnisse vorgestellt. Abschließend fasse ich die Erträge der Studie kurz zusammen.

\section{Kulturen als Diskursfelder}

,Kultur' ist ein Begriff, der in vielen Bedeutungsvarianten abgrenzbare soziale Zusammenhänge von symbolischen Ordnungen, Artefakten und Praktiken bezeichnet. Traditionellerweise ist dieser Begriff mit Konnotationen des Statisch-Homogenen versehen, bspw. als Habitus des Bildungsbürgertums, der deutschen Kulturnation oder als ,Lebensweise und Weltsicht' der Trobriand-Insulaner. Marshall Sahlins (1981) bspw. begreift im Anschluss an strukturalistisch-semiotische Perspektiven 
Kultur als eine spezifische sozial-räumliche Verknüpfung von Deutungs- und Handlungssystemen, als je besonderer, stabilisierter Ausschnitt aus dem unendlichen Möglichkeitsraum gesellschaftlicher Weltbezüge. Die konkrete Entfaltung menschlicher Bedürfnisse und Interessen ist kulturell und damit natürlich sozial strukturiert. Aus einer anderen, hermeneutisch-interpretativen Tradition heraus konzipierte auch Clifford Geertz (1973) in seinen „Dichten Beschreibungen“ abgrenzbare soziale Kollektive als kulturelle Einheiten, als Träger und Nutzer eines homogenen Bedeutungsgewebes, in das sie verstrickt sind. Kultur wird sowohl bei Sahlins wie bei Geertz - respektive in den Traditionen, für die sie stehen - trotz der sehr unterschiedlichen theoretischen Zugänge als stabiles, tradiertes, den Akteuren vorgegebenes Signifikationssystem analysiert. Doch wie kommt ,Kultur' selbst als unterscheidbare, abgrenzbare Einheit zustande? Wie ist es tatsächlich mit ihrer Statik und Homogenität bestellt? Tatsächlich und unweigerlich sind statisch-uniform konnotierte Kulturbegriffe, ob sie nun in der strukturalistisch-semiotischen oder in der hermeneutisch-interpretativen Perspektive formuliert wurden, in den letzten Jahrzehnten zunehmend in die Kritik geraten. Dies gilt sowohl für den westeuropäisch geprägten Blick auf die vorgeblich eingeforene Starrheit ,kalter', nicht-moderner Stammesgesellschaften, wie auch - und vielleicht noch mehr - für die Analyse moderner, globalisierender Großgesellschaften (Clifford 1992; Reckwitz 2000).

Die angesprochene Dynamisierung des Kulturbegriffs kann an wissenssoziologische und diskurstheoretische Traditionen anschließen, die seit langem die gesellschaftliche Produktion von Wissen, d.h. Deutungsvorräten und Handlungsmustern theoretisch zu denken und empirisch zu untersuchen bemüht sind. ${ }^{1}$ Kultur ist dann als dynamisches, konflikthaftes „Diskursfeld“ (Schiffauer 1995) zu begreifen, d.h. als permanenter Aushandlungsprozess von symbolischen Ordnungen, Artefakten und angemessenen Praktiken, der in unterschiedlichen sozialen Arenen, auf verschiedenen gesellschaftlichen Ebenen, unter Beteiligung heterogener gesellschaftlicher Akteure geführt wird und nur vorübergehend sozial-räumlich feste Muster oder Strukturen kristallisiert. Dazu muss Kultur analytisch präzisiert werden: als Begriff, der Deutungs- und Handlungsmuster nicht als frei flottierende Größen fasst, sondern als gebunden an soziale Strukturierungsprozesse, eingeschrieben in die Institutionen, in Handlungen wirkend und dadurch in der gesellschaftlichen Wirklichkeit verankert. ${ }^{2}$ "Culture in action“ (Swidler 1986) ist ein dynamisches Konfliktfeld, in dem Stabilisierungen kontingenten Charakter haben und nur auf Zeit erfolgen.

1 Etwa im Symbolischen Interaktionismus, der phänomenologischen Wissenssoziologie, der Foucaultschen Diskurstheorie sowie neueren diskurstheoretischen Entwicklungen (vgl. Keller 1997b, 2001; Keller u. a. 2001).

2 Vgl. mit Bezug auf den Ideologiebegriff Boltanski/Chiapello (1999, 35). 
Gesellschaften unterscheiden sich kulturell dann und insoweit, wie sie unterschiedliche Deutungs- und Handlungsmuster und in gewisser Hinsicht auch Artefakte für vergleichbare Phänomenbereiche diskursiv-institutionell stabilisieren. Beispielsweise ist ,Natur' keine objektiv gegebene Größe, sondern durch gesellschaftliche Institutionen, Praktiken und Diskurse symbolisch-materiell mitkonstituiert. Das betrifft das erzeugte Wissen über Prozesse, Zusammenhänge und Wechselwirkungen, gesellschaftliche Klassifikationssysteme, auf deren Grundlage institutionelle Regulierungsprozesse ablaufen, die Aushandlung von ästhetischen und moralischen Wertmaßstäben ebenso wie die Schaffung von Artefakten zur produktiven oder konsumtiven Naturnutzung und -abwehr. In modernen Gesellschaften sind kollektive Akteure in vielfacher Weise in Interpretationskonflikte über die angemessene Definition des symbolischen Weltbezugs eingebunden. ${ }^{3}$ Dabei geht es um Bestimmungen dessen, was faktisch der Fall ist, um Fragen der Verantwortungszuschreibung sowie um die Formulierung von Lösungsmöglichkeiten und Folgen. Kultur als Diskursfeld zu begreifen, impliziert, auf Auseinandersetzungen um Reproduktion und Veränderung von Deutungsweisen und Handlungspraktiken hinzuweisen, die vielgestaltige Strukturiertheit, (Re-)Produktion, Heterogenität und Wandelbarkeit soziokultureller Ein- oder besser: ,Vielheiten', die Bedeutung der diskursiven Artikulationskämpfe für die Erzeugung, Identitätsstabilisierung und Transformation solcher Diskurs-Kollektive zu betonen.

\section{Diskurs}

\subsection{Diskurstheoretische Grundlagen}

In der konzeptionellen Anlage der weiter unten vorgestellten Untersuchung wurde ein Ansatz verfolgt, der sich als Wissenssoziologische Diskursanalyse bezeichnen lässt und unterschiedliche wissenssoziologische Perspektiven aufeinander bezieht (Keller 2001): Überlegungen zur "gesellschaftlichen Konstruktion der Wirklichkeit“, die Peter Berger und Thomas Luckmann in den 60er Jahren entwickelt haben (Berger/ Luckmann 1969); Vorschläge zur „frame“ (Rahmen)-Analyse öffentlicher Diskurse, wie sie in der US-amerikanischen Bewegungsforschung vor allem von William Gamson u. a. gemacht wurden (z. B. Gamson/Modigliani 1989); und schließlich allgemeinere diskurstheoretische Überlegungen von Michel Foucault (Foucault 1974a,b; 1981). Während sich Berger/Luckmann aus handlungstheoretischer Perspektive für den interaktiven Aufbau, die gesellschaftliche Objektivierung und sub-

3 Vgl. zur, Culture of public problems` am Beispiel der, Trunkenheit am Steuer' klassisch Gusfield (1981). 
jektive Aneignung von gesellschaftlichen Sinnordnungen interessierten, betonte Foucault in seinen Arbeiten die Emergenz solcher Wissensordnungen, die ihnen zugrundeliegenden Aussageformen und formativen Regeln sowie die mehr oder weniger eigendynamische Rolle sozialer Praktiken. Die Foucaultsche Diskurstheorie sensibilisiert auch für die Bedeutung von Macht, für institutionelle Strukturierungen von Sprecherpositionen und legitimen Inhalten, d. h. für Diskurse als strukturierte und strukturierende Strukturen (Bourdieu 1993, 98). Im Symbolischen Interaktionismus und der wissenssoziologischen Tradition von Berger/Luckmann rückt die interaktive Grundlage, dialektische Gestalt und Prozesshaftigkeit der „gesellschaftlichen Konstruktion der Wirklichkeit" sowie deren Verankerung auch auf der Ebene gesellschaftlich-öffentlicher Definitionskonflikte in den Mittelpunkt. Auch wurden in diesem interpretativen Paradigma in den letzten Jahrzehnten nützliche hermeneutisch reflektierte Verfahren qualitativer Sprach-, Bedeutungs- und Textanalyse auf einem vergleichsweise elaborierten Niveau entwickelt (Keller 2003a).

Diskurse sind abgrenzbare Bedeutungsarrangements, die in spezifischen Sets von Praktiken (re)produziert und transformiert werden. Sie erscheinen in Gestalt einzelner diskursiv-praktischer Ereignisse. Sie ,existieren' als relativ dauerhafte und regelhafte, d.h. zeitliche und soziale Strukturierung von (kollektiven) Prozessen der Bedeutungszuschreibung. Sie werden durch das Handeln von sozialen Akteuren ,real', stellen spezifisches Wissen auf Dauer und tragen umgekehrt zur Verflüssigung und Auflösung institutionalisierter Deutungen und scheinbarer Unverfügbarkeiten bei. Ohne Sprecher, Akteure, Artikulierende oder praktisch Handelnde ,existieren' Diskurse also nicht. Wenn davon gesprochen werden kann, dass Diskurse Themen als gesellschaftliche Deutungs- und Handlungsprobleme kristallisieren oder konstituieren, so ist dies nur eine sprachliche Kurzformel dafür, dass soziale Akteure, die mehr oder weniger unabhängig voneinander weitgehend identischen Regeln des Sprachgebrauchs folgen, in ihren (Sprach-)Praktiken die entsprechenden Weltinterpretationen prozessieren. Sie tun dies immer im Kontext historischer vorgängiger gesellschaftlich-kollektiver Wissensvorräte, also Typisierungen bzw. Institutionen (im Sinne von Berger/Luckmann 1969). ${ }^{4}$ Inwieweit die in Diskursen prozessierten Deutungsangebote gesellschaftliche Geltungskraft erlangen, vielleicht sogar zur fraglosen Wirklichkeit avancieren, und aufgrund welcher Mechanismen und Ressourcen dies geschieht, ist eine empirische Frage. Der Wissenssoziologischen Kultur- als Diskursanalyse geht es darum, Prozesse der sozialen Konstruktion, Objektivation, Kommunikation und Legitimation von Sinn-, d. h. Deutungs- und Handlungsstrukturen auf der Ebene von Institutionen, Organisationen bzw. sozialen Akteuren nicht als singuläre Aussage-Ereignisse, sondern als strukturierte Zusam-

4 Wenn nachfolgend mitunter davon gesprochen wird, eine Diskurs ,tue" dies oder das, so ist dies immer ein Kürzel für die erwähnten Zusammenhänge. 
menhänge, d. h. eben: als Diskurse zu rekonstruieren und die gesellschaftlichen Wirkungen dieser Prozesse zu analysieren. Das schließt unterschiedliche Dimensionen der Rekonstruktion ein: Modi der Bedeutungsproduktion ebenso wie Handlungspraktiken, Artefakte, institutionelle, strukturelle und materielle Kontexte sowie gesellschaftliche Folgen.

Öffentliche Diskussionsprozesse über gesellschaftliche Probleme können als Diskurse begriffen und im Hinblick auf ihre formalen und inhaltlichen Strukturen untersucht werden. Im Wesentlichen geht es dabei um die Annahme institutionell gestützter Selektionskriterien für die Beteiligung an Diskursen - Wer darf legitimerweise wo sprechen? -, für die formulierbaren Inhalte - Was darf/kann gesagt werden? -, um die Regeln der Artikulation - Wie kann etwas gesagt werden? - sowie um die dabei eingesetzten Ressourcen. Darüberhinaus betont eine diskursorientierte Perspektive die weltkonstituierenden Effekte von Aussagezusammenhängen im Sprachgebrauch. Die Welt gewinnt ihren je spezifischen Wirklichkeitscharakter für uns durch die Aussagen, die Menschen - in Auseinandersetzung mit ihr - über sie treffen, wiederholen und auf Dauer stellen. Solche Aussagen stiften nicht nur die symbolischen Ordnungen und Bedeutungsstrukturen unserer Wirklichkeit, sondern sie haben auch reale Konsequenzen: Gesetze, Statistiken, Klassifikationen, Techniken, Artefakte oder Praktiken.

\subsection{Die Erschließung von Diskursinhalten}

Wissenssoziologische Diskursanalyse bezeichnet ein Forschungsprogramm, aber keine spezifische Methode. Ihre empirische Umsetzung kann deswegen nicht in einem Standardmodell vorgeschrieben werden. Sie ist vielmehr für die jeweiligen Gegenstandsbereiche und Forschungsinteressen je angemessen zu leisten und zu begründen. Das schließt nicht aus, bei vergleichbaren Untersuchungsfeldern und Fragestellungen auch vergleichbare Vorgehensweisen zu wählen. Zur Analyse öffentlicher Auseinandersetzungen als Diskurse schlage ich die Unterscheidung von sozio-kulturellen Deutungsmustern, rotem Faden (story-line, plot) und diskursspezifischem Interpretationsrepertoire vor. Damit sind verschiedene ,Bausteine' bezeichnet, die für die Erschließung der inhaltlichen Strukturierung von Diskursen eingesetzt werden können. Neben dieser auf Inhalte bezogenen Konzepte interessiert sich die soziologische Diskursanalyse natürlich für den Sprachgebrauch bzw. das Aussageereignis als soziales und sozial strukturiertes Phänomen und benötigt dazu einige zusätzliche Kategorien: Zum Kontext eines Diskurses gehören etwa das institutionellorganisatorische Feld, in dem er erscheint, die spezifische (auch: historische) Situation seines Auftretens, andere Diskurse, mit denen er konkurriert oder gegen die er sich richtet. Gesellschaftliche Akteure verwenden unterschiedliche Ressourcen - 
nicht nur Sprache bzw. Zeichen, sondern Geld, Macht, Einfluss, Ansehen, Beziehungen u. a.m. - und tragen dadurch zur (De)Stabilisierung von symbolischen und materiellen Ordnungen bei. Mit dem Begriff des Dispositivs können die institutionalisierten, sprachlichen und materiellen Vergegenständlichungen von Diskursen in ihrer Gesamtheit benannt werden. Diskurseffekte sind nicht nur solche Vergegenständlichungen, sondern auch Subjektpositionen, d. h. Subjektkonzeptionen und daran geknüpfte Handlungsschemata, die im Diskurs formuliert werden. Einzelne Sprachereignisse lassen sich als Diskursfragmente begreifen. Bedeutsam für öffentliche Diskurse sind schließlich die Massenmedien als Arena der Konfliktaustragung mit eigenen Verlaufsmechanismen. Nachfolgend werde ich mich nur mit dem Begriff des Deutungsmusters näher befassen. ${ }^{5}$

Die Konstitution und Aufbereitung des Themas oder Referenzphänomens eines Diskurses erfolgt unter anderem durch die diskursspezifische Verknüpfung allgemeiner Deutungsmuster, die im Kontext einer Wissensgemeinschaft verfügbar sind. Es handelt sich dabei um Schemata, die für individuelle und kollektive Deutungsarbeit im gesellschaftlichen Wissensvorrat zur Verfügung stehen und in ereignisbezogenen Deutungsprozessen aktualisiert werden. Der Begriff des Deutungsmusters visiert den sozial typischen Sinn einer Äußerung an, also eine gesellschaftlich vorübergehend konventionalisierte Deutungsfigur. Deutungsmuster bilden die Schnittstelle zwischen ,Sendern' und ,Empfängern' von Texten. Unabhängig davon, welche Intentionen mit Aussagekomplexen verfolgt werden, lässt sich festhalten, dass ihre Produzenten im Spachgebrauch auf kollektiv verfügbare Deutungsmuster zurückgreifen und dadurch spezifische Interpretationen der Phänomenbereiche nahelegen, auf die Aussagen referieren. Ein Deutungsmuster verknüpft unterschiedliche Bedeutungselemente zu einer kohärenten (nicht notwendig: konsistenten) Deutungsfigur, die in unterschiedlicher manifester Gestalt auftreten kann. Deutungsmuster werden in der wissenssoziologischen Tradition immer schon als kollektive Produkte begriffen, die im gesellschaftlichen Wissensvorrat vorhanden sind und sich in konkreten sprachlichen Äußerungen manifestieren. Es handelt sich um historisch, in Interaktionen ausgebildete Interpretationsmuster der Weltdeutung und Problemlösung, (...) (die) den einzelnen Subjekten gegenüber zwar gesellschaftlich insofern vorgängig sind, als das einzelne Subjekt in ein bereits vorhandenes, historisch ausgebildetes, sprachlich repräsentiertes System von Regelstrukturen, Wissensbeständen und gesellschaftlicher Praxis hineingeboren und sozialisiert wird; doch diese sozialen Strukturen existieren weder unabhängig von den Handlungen der Subjekte noch führen sie ein Eigenleben (.)“ (Lüders/Meuser 1997, 62 f.; vgl. auch Plaß/Schetsche 2001). Sie basieren vielmehr im Sinne der von Anthony Giddens formulierten Kon-

5 Der vorgegebene Rahmen zwingt zu dieser Einschränkung. Vgl. deswegen ausführlicher Keller $(1998,2001,2003 a, b)$. 
zeption der „Dualität von Struktur“ auf den „bewusst vollzogenen Handlungen situierter Akteure, die sich in den verschiedenen Handlungskontexten jeweils auf Regeln und Ressourcen beziehen (...) “ (Giddens 1988, 77 ff.). Diskursanalyse bezieht sich nicht nur auf die Rekonstruktion solcher mehr oder weniger diskursspezifischer Arrangements von Deutungsmustern, sondern auch auf die Prozesse ihrer Formung, Verhandlung und Transformation durch gesellschaftliche Akteure, die in ihrer Diskurspraxis in institutionell-organisatorische Felder, Regeln und Ressourcen, in konflikthafte symbolische Kämpfe eingebunden sind. ${ }^{6}$

Als deutungsgenerierende Schemata schwimmen Deutungsmuster selten auf der expliziten Oberfläche der Aussagen und Texte. Sie werden auf der Ebene von textförmig zugänglichen Aussageereignissen in einem hermeneutisch reflektierten Interpretationsprozess rekonstruiert, ${ }^{7}$ der verschiedene Analysestufen umfasst: Zunächst wird nach (theoriegeleiteten) Kriterien das Textkorpus zusammengestellt, das als Manifestation des gesamten Diskurses der Untersuchung zugrunde liegt. Dazu ist ein gewisses Vorwissen über das Untersuchungsfeld notwendig, das gleichwohl nicht zu spezifischen Blickverengungen, also Vorurteilen bei der Dokumenten- bzw. Datenauswahl führen sollte. Dann werden im Durchgang durch das Datenkorpus spezifische Daten (Dokumente) für die Feinanalyse ausgewählt, wobei der Auswahlprozess nach Maßgabe der ersten Feinrekonstruktionen modifiziert werden kann. Als Auswahlstrategien stehen unterschiedliche Relevanzkriterien zur Verfügung: im Feld benannte Schlüsseltexte, Merkmale minimaler und maximaler Kontrastierung u. a.m. Nach Maßgabe der allgemeinen Fragestellungen einer Untersuchung werden einzelne Textpassagen oder ganze Texte für eine interpretierende Satz-für-Satz-Analyse ausgewählt, bei der im Fortgang der Textauslegung Deutungshypothesen entworfen, am Text geprüft, mitunter verworfen, verfeinert oder ersetzt werden, bis schließlich die ,adäquateste' Deutung gefunden ist. Diese Prozedur wird im Durchgang durch die verschiedenen ausgewählten Dokumente wiederholt, bis schließlich ein Eindruck der Sättigung entsteht, also weitere Analysen keine neuen Deutungen mehr ergeben. Die Ergebnisse der einzelnen Feinanalysen müssen dann kombinatorisch aufeinander bezogen werden. Die erwähnten Untersuchungsschritte sind eingebunden in einen weiteren Forschungszusammenhang, der auch die Erhebung von Kontextdaten, die Modifikation von Forschungsfragen und Hypothesen im Untersuchungsprozess, die Auseinandersetzung mit anderen Forschungsergebnissen über den Untersuchungsgegenstand und Prozesse der abschließenden Gesamtinterpretation bzw. Theoriebildung einschließt. ${ }^{8}$

Diskurse bauen auf mehreren Deutungsmustern auf. Die Analyse solcher Deutungsmuster, ihrer argumentativen oder narrativen Verknüpfungen und symbo-

6 Vgl. dazu auch die methodischen Hinweise bei Lüders/Meuser (1997, 67 ff.).

7 Vgl. Hitzler/Honer (1994); Soeffner/Hitzler (1994); Hitzler/Honer (1997). 
lisch-rhetorischen Manifestationen führt zum diskursspezifischen Interpretationsrepertoire. Es enthält die Bausteine, die innerhalb eines Diskurses „für die Interpretation von Handlungen, der eigenen Person und gesellschaftlicher Strukturen im Sprechen verwendet werden" (Potter/Wetherell 1995, 188 f.). Dazu zählen neben den Deutungsmustern auch Oberflächenstrukturen der Äußerungen, bspw. häufig verwendete Bilder (Metaphern) oder kognitive Strukturierungen (z. B. Klassifikationen). Diese Bausteine werden im jeweiligen Diskurs durch einen roten Faden, eine story-line (plot) zu einer besonderen ,Erzählung' zusammengeführt und auf einen Anlass bezogen. Story-lines verbinden die unterschiedlichen Deutungsmuster und bilden dadurch die narrative Struktur eines Diskurses. Sie liefern die ,Handlungsschemata' für die Erzählung, mit der sich der Diskurs erst an ein Publikum wenden kann (Keller 1998, 36 ff.; Viehöver 2001, 2003). Solche narrativen Strukturen liegen selbst ,trockenen', abstrakten wissenschaftlichen Texten zugrunde, auch wenn wir sie mitunter dort kaum vermuten.

\subsection{Die Massenmedien als öffentliche Diskursarena}

Die Massenmedien stellen einen öffentlichen Raum für Diskurse zur Verfügung. Erst die Repräsentation in den Massenmedien stiftet in den Gegenwartsgesellschaften letztlich die Qualität eines öffentlichen Diskurses. Themenbezogen manifestieren sich dabei Diskurse, die ihren Entstehungsort nicht (nur) in den Medien haben, die aber auf der Grundlage der Medienberichterstattung als öffentliche Diskurse empirisch rekonstruiert werden können. Die in den Massenmedien erzeugten Texte sind Beiträge zur gesellschaftlichen Wirklichkeitskonstruktion. Medien,arbeiter' produzieren nicht unbedingt besondere Folien zur Interpretation von Wirklichkeit, vielmehr schöpfen sie aus dem gesellschaftlichen Wissensvorrat in ähnlicher Weise wie andere Akteure. Allenfalls können ihnen spezifische Fokussierungsleistungen zugerechnet werden. Texte, die darin erscheinen, werden nicht nur spezifischen Textformaten/ Textgattungen angepasst - z. B. als Nachricht, Kommentar, Hintergrundbericht -, sondern ihnen liegen auch verschiedene Selektionsstufen und -prozesse wie Routinen der Berichterstattung, Nachrichtenwerte, professionelles agenda-buildung und vermutete Resonanzfähigkeit zugrunde. ${ }^{9}$ Die Bedeutung ,kultureller Resonanzen' für die Diskursrezeption wurde von David Snow, William Gamson u. a. im Kontext der US-amerikanischen Forschung über Mobilisierungsprozesse sozialer Bewegun-

8 In der qualitativen Sozialforschung, aus der diese Analysestrategien stammen, lauten die entsprechenden Konzepte: Sequenzanalyse, rekonstruktive Hermeneutik, grounded theory, Kodieren, Triangulation u. a.m. (vgl. als ausführliche Erläuterung Keller 2003a).

9 Vgl. ausführlicher zur Bedeutung und Analyse massenmedialer Diskurse Keller (1997a und 1998). 
gen betont (vgl. etwa Gamson/Modigliani 1989). Gemeint ist damit, dass die Akteure/Diskurse günstigerweise diskurspezifisch solche Interpretations- bzw. Deutungsmuster einsetzen, die in einem weiteren gesellschaftlich-kulturellen Wissensvorrat (Kontext) mit besonders starker Beachtung, Wertbindungen, Affekten usw. besetzt bzw. von breiten gesellschaftlichen Gruppen akzeptiert sind. In der bundesdeutschen Nachkriegsdebatte zählen dazu bspw. die Idealisierungen der ,freien Marktwirtschaft' oder die Warnung vor ,kommunistischer Gefahr', aber auch die starke Vorstellung, es dürfe hierzulande ,nie wieder Faschismus' geben. Poferl (1997) liefert ein schönes Beispiel solcher Resonanzstrategien am Beispiel der Tschernobyl-Berichterstattung in der FAZ.

Genau genommen handelt es sich bei massenmedialen Diskursen - bspw. über Abfallprobleme - um solche Teildiskurse innerhalb umfassenderer Diskursformationen, die gerade die öffentliche Sinnhaftigkeit eines Themas konstituieren. Die im Medienbetrieb produzierten Texte strukturieren die Relevanzmuster menschlichen Deutens und Handelns auf den unterschiedlichsten Ebenen. Medienberichterstattung ist Teil des permanenten Prozesses der Festschreibung oder Veränderung des Bedeutungsgewebes ,Kultur' (Burgess 1990, 143). Die Massenmedien bestimmen durch ihre Selektionsprozesse etwa den kulturellen Code des Politischen mit, d.h. „was politisch denkbar ist“, und wer „zu den legitimen Akteuren des politischen Spiels zählt" (Bourdieu 1992, 88). Sie sind zugleich Bühne und Protagonist der öffentlichen Diskurse, beobachten und kommentieren die aufeinanderbezogene Rede der Akteure und veröffentlichen sie spezifisch gefiltert. Die Massenmedien eignen sich damit in besonderer Weise als Grundlage für eine empirische Analyse öffentlicher Diskurse. Sie sind Arenen, in denen über die (öffentliche) Bedeutung von ,Botschaften`entschieden wird.

\section{Der Hausmüll zwischen Katastrophismus und Beherrschungsritualen}

Der im vorangehenden Kapitel skizzierte Ansatz der Wissenssoziologischen Diskursanalyse lag einer vergleichenden Untersuchung öffentlicher Diskussionen über das ,Hausmüllproblem ' in Deutschland und Frankreich zugrunde. Beide Länder sahen sich erstmals Anfang der 1970er Jahre und dann bis Mitte der 1990er Jahre mit Anforderungen zum Auf- und Ausbau landesweiter Abfallgesetzgebungen konfrontiert, die von einer mehr oder weniger breiten öffentlichen Diskussion begleitet wurden. Im Rahmen der Studie wurde ein umfangreiches Datensample aus Texten der Printmedien - je etwa 700 Artikel unter Berücksichtigung des Meinungsspektrums - sowie aus abfallpolitischen Dokumenten und Expertenmaterialien für den Zeitraum der abfallpolitischen Debatten und Gesetzgebungen von 1970-1995 zusammengetragen. Zusätzlich wurden in Deutschland und Frankreich einige Exper- 
teninterviews mit Protagonisten und Beobachtern der Diskussionen geführt. Die nachfolgend auszugsweise vorgestellten Ergebnisse einer qualitativen, hermeneutisch-interpretativ ansetzenden Rekonstruktion der inhaltlichen Strukturierung der jeweiligen Abfalldebatten basieren auf einer theoriegeleiteten Auswahl von Dokumenten aus dem Printmediensample. ${ }^{10}$

\section{1 Überblick: Abfall in Deutschland und Frankreich}

Alle wesentlichen technischen, administrativen und infrastrukturellen Elemente unseres heutigen Umgangs mit Abfall stammen aus dem 19. Jahrhundert: Mülltonnen, kontrollierte Deponien, Müllverbrennung, technisierte Müllverwertung. Das ist die Zeit der Erfindung des modernen Umgangs mit Müll - und der Zerstörung der vormodernen Praktiken (Keller 1998). In den 1960er Jahren beschleunigen die Unternehmen aus absatzstrategischen Gründen den Güterumsatz; steigende Geldvermögen der Haushalte erweitern gleichzeitig die Konsumspielräume. Wegwerfkonsum und eine beschleunigte Vermodung von Gebrauchs- wie Luxusgütern sind Bestandteile des stattfindenden kulturellen Trainings. Die entstehenden Supermärkte wurden zu repräsentativen Orte der neuen Konsumkultur. Mit der Wohlstandssteigerung und den sich verändernden Konsummustern kommt es in Deutschland und Frankreich zu wachsenden Problemen der Abfallbeseitigung. Die vorhandenen Entsorgungsinfrastrukturen - in erster Linie Deponien - können mit dem anfallenden und prognostizierten Müllwachstum nicht mithalten. Der Umstellung auf billige Massenproduktion und Einwegprodukte folgt die gesellschaftliche Praxis des ,wilden Müllens', der allgegenwärtigen anarchischen Abfallentsorgung. Die Sorge der Kommunen verbindet sich mit der Empörung der Bevölkerung und der skandalisierenden Berichterstattung der Lokalpresse. Schließlich steht Ende der 1960er Jahre sowohl in der Bundesrepublik Deutschland als auch in Frankreich die landesweite Neuregelung der Abfallentsorgung auf der politischen Tagesordnung. Die jeweiligen Regierungen nehmen sich der Sache an.

Auch wenn bis dahin von einer gemeinsamen Ausgangslage der deutschen und der französischen Abfallsituation gesprochen werden kann, entwickeln sich die jeweiligen Politikfelder nun sehr unterschiedlich - zumindest, was ihre öffentliche und diskursive Repräsentation angeht. In Deutschland entfaltet sich die Mülldiskussion zur Chronik einer angekündigten Katastrophe; in Frankreich dagegen handelt es sich um die ritualistische Chronik eines angekündigten zivilisatorischen Sieges. In der Bundesrepublik konkurrieren in der massenmedial vermittelten Öffentlichkeit

10 Vgl. zur Vorgehensweise und Ergebnissen die Hinweise weiter oben in Kapitel 2.2 und ausführlich Keller (1998, 2003b). 
zwei Diskurse mit sehr unterschiedlichen Deutungsmustern um die Definition der Abfallverhältnisse. In den französischen Medien ist dagegen nur ein Abfalldiskurs repräsentiert. Damit sind zwei differierende öffentliche Ordnungen legitimer Äußerungen und Akteure konstituiert. Nur in Deutschland kommt es zur breiten, öffentlichen Thematisierung einer Abfallpolitik, die nicht ,end of pipe', sondern auf die Produktkultur selbst hin orientiert ist. Anfang der 90er Jahre werden abfallpolitische Regulierungen eingesetzt, deren Grundzüge bereits 1970/71 von Experten vorgeschlagen worden waren (vor allem: Verpackungsverordnung). Hier kommen zudem höhere (rohstoffliche) Verwertungsquoten, Abfallgebühren (im Verhältnis 3:1) und schärfere Grenzwerte zum Einsatz. Die Umsetzung der Abfallpolitik erfolgt zügiger als in Frankreich. In der Tendenz setzen beide Länder zunehmend auf getrennte Sammlung und Verwertung von Abfällen, auf einen Ausbau der Müllverbrennung und auf hohe Sicherheitsstandards für wenige verbleibende Restmülldeponien. Unter dem neuen, die Versöhnung von Ökonomie und Ökologie suggerierenden Leitbild der ,Nachhaltigkeit' soll eine Kombination aus optimierten Entsorgungs- und Verwertungstechnologien, Produktoptimierungen und Selbstverpflichtungen der Industrie die Abfallprobleme dauerhaft lösen. ${ }^{11}$

Während in der Bundesrepublik Deutschland zunächst die Abfallbeseitigung, dann jedoch - im Hinblick auf Umweltverschmutzung und globale Rohstoffknappheit - die Abfallverwertung und Kreislaufführung der Abfälle im Vordergrund steht, hatte in Frankreich im Hinblick auf nationale Rohstoffknappheit von Beginn an der ,Kampfgegen die Verschwendung' durch Abfallverwertung programmatisch Priorität. In Deutschland verläuft die Abfalldiskussion im Schatten der angekündigten Müllkatastrophe: das Land, vielleicht die Welt werden im Müll ersticken, wenn nicht sofort gehandelt wird. In Frankreich hingegen handelt es sich von Beginn an um ein staatlich angekündigtes, gleichwohl im Verlauf der Diskussion nicht eingelöstes Beherrschungsversprechen bezüglich des Müllaufkommens.

Zu Beginn der 1970er Jahre, in der ersten Phase der neueren Abfallpolitik, setzt man in beiden Ländern auf technisch optimierte Abfallbeseitigung - etwa höhere Schornsteine, Deponienormierung - bzw. in Deutschland weniger, in Frankreich stärker, auf die Förderung von Recycling. Technischer Fortschritt gilt als probates Mittel der Beseitigung der Nahfolgen. Danach geht die Entwicklung unterschiedliche Wege: In Frankreich bleibt es zunächst bei der Thematisierung von Nahfolgen, des sichtbaren Mülls. In Deutschland werden in den 1980er Jahren mit der Dioxinproblematik zunehmend definierte Fernfolgen der Müllbeseitigung diskutiert. Um das Hausmüllthema konstituiert sich eine breite Bürgerbewegung, die Müllbeseitigungstechnologien unter einen generellen Risikoverdacht stellt. Zunehmend entstehen Abfallwirtschaftskonzepte und Recyclinginitativen ,von unten'. Darauf wird

11 Vgl. zu den einzelnen Stationen des jeweiligen Regulierungsprozesses Keller (1998). 
politisch auch, aber nicht nur, mit neuer technischer Normierung der Entsorgungsstandards reagiert. Zusätzlich werden im Kontext der Nachhaltigkeitsdiskussion Produktentwicklung und Konsumformen in globalisierten Verbrauchsparametern verortet. Die vergleichsweise weit ausholenden französischen Bemühungen der Abfallgesetzgebung Anfang der 1990er Jahre lassen sich als Antizipation und Import entsprechender (angekündigter) transnationaler Rechtsnormen verstehen.

\subsection{Die bundesdeutsche Abfall-Öffentlichkeit als Streitarena}

Im Vergleich der öffentlichen Diskussionen über die richtige Müllpolitik in Deutschland und Frankreich wird die Rolle der Definitionsverhältnisse deutlich. In der Bundesrepublik streiten in der massenmedial vermittelten Abfalldiskussion von Beginn an zwei Diskurse um die legitime Interpretation des Abfallproblems: Experten und Medien verstehen sich hier auch als Anwälte kritischer Interessen schon Ende der 1960er Jahre - noch vor dem Entstehen der entsprechenden sozialen Bewegungen. Typisierend lassen sich die beiden erwähnten Diskurse benennen als strukturkonservativer Diskurs technisch-ökologischer Modernisierung und als (herausfordernder) kulturkritischer Diskurs politisch-ökologischer Restrukturierung. Sie unterscheiden sich durch ihre Deutungen des Hausmüllproblems (vgl. Abbildung 1) und werden von unterschiedlichen Diskurskoalitionen getragen, deren Zusammensetzung sich im Verlauf der Diskussion verändert (Keller 1998, 270 ff.). Die öffentliche Resonanz des herausfordernden Abfalldiskurses bringt den strukturkonservativen Diskurs, der die materiale Bundesabfallpolitik dominiert, soweit in praktische Bedrängnis und Legitimationsnot, dass Ende der 80er Jahre grundsätzlich neue Ansatzpunkte der Abfallpolitik ausgelotet werden müssen.

Welche Interpretationen des Hausmüllproblems werden nun in den erwähnten Diskursen vorgetragen? Welche Deutungsmuster spielen dabei eine Rolle? ${ }^{12}$ Für den strukturkonservativen Diskurs entsteht das Abfallproblem aus der Diskrepanz zwischen verfügbaren Anlagen der Abfallbehandlung und dem Abfallaufkommen. Abfallentstehung ist demnach eine unabänderliche Begleiterscheinung von Wohlstand, Massenkonsum und Fortschritt. Die vernünftige Problemlösung besteht im Ausbau der fehlenden technischen Infrastruktur. Die modernen Anlagen der Abfallbehandlung gelten als sicher und umweltverträglich. Staatliches Handeln soll sich auf ein Minimum an Rahmenbedingungen konzentrieren. Weitere Eingriffe in die Produktionsentscheidungen der Unternehmen sind nicht notwendig und gefährden den Wirtschaftsstandort Deutschland. Im Kern werden hier Deutungsmuster verknüpft, die die Autonomie des Marktes (der Wirtschaft) an die erste Stelle setzen,

12 Vgl. zur Vorgehensweise weiter oben Kapitel 2.2. 
das Abfallaufkommen als ein sich naturwüchsig ergebendes und dann technisch handhabbares Problem begreifen. Natur gilt als unerschöpfliche Ressource; die Position sieht sich selbst als verantwortungsethisch angemessen - gegen den blinden, irrationalen Eifer der Gesinnungsethiker. Der Diskurs favorisiert einen sachlichnüchternen, technikorientierten Stil. Darin gleicht er seinem französischen Pendant (s.u.). Er hat seinen massenmedialen Ort in Zeitungen wie der FAZ oder Der Welt, dem Handelsblatt usw. Träger sind die CDU, die FDP, Teile der SPD, große Teile der Wirtschaft u. a.m.

Spiegelbildlich dazu entwirft der alarmistische kulturkritische Abfalldiskurs die folgende Deutung der Abfallsituation: Die, Wegwerfgesellschaft' betreibt einen unmäßigen Stoffverbrauch, der im Nord-Süd-Vergleich ethisch-moralisch ungerechtfertigt, im Hinblick auf die Begrenztheit der Ressourcen des Planeten und seiner Funktion als Schadstoffsenke auch ökologisch unvernünftig, unverantwortlich und unmoralisch ist. Verantwortlich dafür sind die Produktions- und Absatzstrategien der Wirtschaft, die mit den Mitteln beständiger Bedürfniserzeugung, Produktersetzung, der Umstellung auf Einweg-Produkte u. a.m. die gesellschaftliche WegwerfKultur erzeugt. Verantwortlich ist auch die Politik, da sie der Wirtschaft nicht die entsprechenden Rahmenbedingungen für eine veränderte Stoffnutzung setzt. Von den existierenden Abfallbehandlungsanlagen gehen Gefährdungen für menschliche Gesundheit und die Umweltmedien (Luft, Wasser, Böden) aus, die in ihren Folgen kaum kalkulierbar und unakzeptabel sind. Technische Problemlösungen (auch Recyclingtechnologien) sind nur Hilfsmittel einer grundsätzlich nötigen politischen Umsteuerung der Produktions- und Konsumverhältnisse. Erst die Neugestaltung der Rahmenbedingungen der Produktion führt von der Wegwerf-Gesellschaft hin zu einer ,abfallarmen' Gesellschaft. Ausgangsprämisse der Argumentation ist hier die Annahme der Knappheit von Natur als Ressource und Schadstoffsenke. Abfallerzeugung gilt als gesellschaftlicher Prozess, der auf Entscheidungen beruht und der damit politisch reguliert werden kann und muss. Technische Abfallbehandlung steht unter einem generalisierten Risikoverdacht. Auch dieser Diskurs reklamiert für sich eine allgemeine verantwortungsethische Position, hier allerdings gegenüber partikularen Profitinteressen. Massenmedial vermittelt wird er etwa im Spiegel, Der Zeit, der taz, der Süddeutschen Zeitung, der Frankfurter Rundschau usw. Träger sind die Umweltverbände, Teile der SPD, Die Grünen, einige Firmen, verschiedene Wissenschaftlergruppen u. a.m. 


\begin{tabular}{|l|l|}
\hline $\begin{array}{l}\text { kulturkritischer Abfalldiskurs: } \\
\text {,politisch-ökologische Restrukturierung }\end{array}$ & $\begin{array}{l}\text { strukturkonservativer Abfalldiskurs: } \\
\text {,technisch-ökologische Modernisierung }\end{array}$ \\
\hline - Politisierung des Wirtschaftens & - Autonomie der Wirtschaft \\
- Vergesellschaftung des Problems & - (Quasi-)Naturalisierung des \\
- Wechsel des Entwicklungsmodells & Problems \\
- Risiko & - Kontinuität des Fortschritts \\
- knappe Natur & - Technisch-administrative Kontrolle \\
- Verantwortungsethik vs.- & - unerschöpfliche Natur \\
Profitinteresse & - Verantwortungsethik vs. \\
& Gesinnungsethik \\
\hline
\end{tabular}

Abbildung 1: Diskurse und ihre Deutungsmuster in der deutschen Hausmülldiskussion ${ }^{13}$

Exemplarische Erläuterungen ausgewählter Deutungsmuster:

\section{(1) Strukturkonservativer Abfalldiskurs}

\section{Autonomie der Wirtschaft}

Dieses Deutungsmuster setzt eine gesellschaftsinterne Trennung zwischen Bereichen der kollektiven (politischen) Gestaltbarkeit und Bereichen der individuellen (unternehmerischen) Gestaltbarkeit (d.h. kollektiven Nichtgestaltbarkeit). Diese konstituiert sich über die Unterscheidung gesellschaftlicher Subsysteme mit autonomen Funktionslogiken und Zuständigkeiten - Wirtschaft (Autonomie ,freier Marktwirtschaft', Befriedigung materieller Bedürfnisse) und Politik (Gestaltung des Zusammenlebens). Die Funktionslogik der, Wirtschaft bestimmt die Grenzen des Gestaltungsspielraums von Politik. Ob die ,Freiheit der Märkte', die ,Freiheit der Bedürfnisse‘, die ,Folterinstrumente der Regierung' oder die ,Gefährdung des Wirtschaftsstandorts Deutschland ${ }^{\star}$ - um nur einige gängige Formeln aufzugreifen beschworen wird, immer geht es darum, ein spezifisches Gesellschaftsmodell der Organisation des Verhältnisses von Wirtschaft und Politik (Administration) aufrecht zu erhalten.

13 Die immer mehr oder weniger glückliche Benennung von Deutungsmustern kann mitunter auf Begriff der analysierten Texte selbst zurückgreifen; meist muss sie eigene Etikette entwickeln. Die Erläuterungen dienen der Nachvollziehbarkeit dessen, was gemeint ist. Ausführlichere Textbeispiele und Erläuterungen der Deutungsmuster finden sich in Keller (1998), exemplarische Beispiele auch in Keller (2003b). 


\section{(Quasi-)Naturalisierung}

Mit dem Begriff der (Quasi-)Naturalisierung ist ein soziokulturelles Deutungsmuster angesprochen, das (soziale) Phänomene auf als außergesellschaftlich gedachte Naturprozesse zurückführt. Bezieht man dieses Interpretationsschema auf gesellschaftliche Prozesse - hier am Beispiel des Abfallaufkommens -, dann entspricht ihm der Verweis auf unverfügbare systemische Größen (wie Modernisierung, Fortschritt, Marktlogik, Bedürfnisentwicklung). Gesellschaft tritt sich darin selbst als ,zweite Natur' gegenüber und reagiert darauf mit den ,klassischen' Mustern industriegesellschaftlicher Naturbegegnung (Gefahrenabwehr und Ausbeutung).

\section{(2) kulturkritischer Abfalldiskurs}

\section{Knappe Natur}

Das zentrale Deutungsmuster der knappen Natur steht für die Idee der absoluten, prinzipiellen Begrenztheit von Natur als Rohstoffressource und Schadstoffsenke. Beide Merkmale lassen sich nicht durch technischen Fortschritt in ihrer Geltungskraft aufheben. Notwendig wird deswegen eine gesellschaftliche Kontrolle der gesellschaftlichen Naturnutzung. Diese muss sich an Prinzipien der Vermeidung und Schonung von Natur, d. h. an der gesellschaftlichen Anpassung an ,natürliche Grenzen' ausrichten. Es bedarf einer gesellschaftlichen Kontrollinstanz, die Langfristinteressen dergestalt wahrnimmt, dass sie in alle Formen der Naturnutzung regulierend eingreift.

\section{Vergesellschaftung}

Der Begriff der Vergesellschaftung bezeichnet ein soziokulturelles Deutungsmuster, das beobachtete (,natürliche` oder soziale) Phänomene auf gesellschaftsinterne und damit als entscheidungsabhängig begriffene, d. h. gestaltbare Prozesse zurückführt. In einer solchen Interpretation werden zwar systemische Prozesse durchaus gesehen, aber gerade die Frage nach ihrer gesellschaftlicher Kontrolle aufgeworfen. Nicht die Abfallbeseitigung, sondern die Abfallentstehung, mehr noch, die rohstoffintensive Dingkultur ist das Handlungsproblem. Der kulturkritische Abfalldiskurs geht von der Idee gelingender gesellschaftlicher Selbstkontrolle aus.

Aus der öffentlichen Konkurrenz und den Mobilisierungsanstrengungen der beiden Diskurse lässt sich die beidseitig unterschiedlich akzentuierte, aber immer hohe Bedeutung des Katastrophischen verstehen - der Müllfluten und Müllberge, in denen wir ersticken werden, der Vergiftungen, die von Behandlungstechnologien ausgehen können, des globalen Endes der Ressource Natur, aber auch der Verstopfung der Entsorgungswege und des Verlustes internationaler Wettbewerbsfähigkeit. ${ }^{14}$ Der

14 Was als Katastrophe gilt, kann mithin sehr unterschiedlich sein. 
kulturkritische Diskurs übernimmt in diesem Wettkampf die Rolle des Herausforderers und Zugpferdes; der strukturkonservative Diskurs folgt ihm nach und integriert zunehmend Teile der gegnerischen Problembeschreibung in sein Interpretationsrepertoire; dieses Zusammespiel beider Diskurse treibt - vermittelt über die strukturkonservative Position - rechtliche und materiell-technische Standards voran. Anfang der 90er Jahre unterscheiden sich die benutzten Problembeschreibungen nur noch in Nuancen. Der Streit ist damit nicht aufgehoben, sondern entzündet sich jetzt an Begriffsinterpretationen und ihren handlungspraktischen Konsequenzen, etwa an der Frage, wann zulässigerweise von Abfallvermeidung gesprochen werden kann oder wie Leitbilder der ,Kreislaufwirtschaft' oder der ,Nachhaltigkeit tatsächlich regulierungs- und handlungspraktisch zu gestalten wären.

\subsection{Die französische Abfall-Öffentlichkeit als Ort hegemonialer Präsentation}

In Frankreich dominiert durchgehend ein etatistischer, administrativ-technischer Kontrolldiskurs die öffentliche Abfalldiskussion. Regelmäßig beklagt er aus seiner hegemonialen Position heraus Defizite der Abfallbeseitigung und kündigt deren baldige Beseitigung an: die französische Zivilisation wird letztlich über die Abfälle triumphieren. Wissenschaftlich-technische Expertise steht - öffentlich unangefochten - im Dienste des Staates und damit des französischen Gesellschaftsprojektes. ,Kritische' Stimmen zur Abfallsituation erscheinen in der massenmedial vermittelten Öffentlichkeit einerseits als journalistische Appelle an den Staat, etwas zu tun (gegen miserable Deponiebedingungen, gegen ausländische Abfallimporte), andererseits und überwiegend als staatliche Vorwürfe an Wirtschaft, Kommunen und Bürger wegen Fehlverhaltens, als Appelle an staatsbürgerliche ,abfallcorrectness', sei es im Hinblick auf Einsparungen bei der nationalen Rohstoffbilanz oder das geforderte ,intelligente' Wegwerfen und Beseitigen. Die Ankündigung einer baldigen technisch-zivilisatorischen Kontrolle der Abfälle trägt Züge eines öffentlichen Rituals der nationalen Selbstvergewisserung und Selbstbeschwörung, der Selbstverpflichtung auf wissenschaftlich-technische Rationalität und zivilisatorische Modernität. Defizite der Abfallpolitik werden auf Einflüsse von außen oder auf mangelnde staatsbürgerliche Disziplin zurückgeführt.

Die Interpretation des Abfallproblems, die der in erster Linie von den Agenturen des Staates getragene technisch-administrative Abfalldiskurs in Frankreich anbietet, lässt sich folgendermaßen zusammenfassen (Keller 1998, 276 ff.): Die in Modernisierungs- und Rationalisierungsprozessen sich verändernden Produktions- und Konsumtionsweisen und der dadurch induzierte materielle Wohlstand führen zu höherem Abfallaufkommen. Dies wird aus zwei Gründen problematisch: Einerseits 
gefährden wilde, unkontrollierte Abfallablagerungen die Umwelt und dadurch auch die menschliche Gesundheit. Sie stellen darüberhinaus eine ästhetische Belästigung (Beeinträchtigung des Landschaftsbildes) dar. Ursache dieser Zustände ist moralisch schuldhaftes, z. T. auch illegales Verhalten (zivil)gesellschaftlicher Akteure (der zuständigen Gebietskörperschaften, der Wirtschaft oder der Bürger), die aus Gründen der Geldeinsparung und Bequemlichkeit gewissenlos möglichst billig ,entsorgen`. Andererseits enthalten die Abfälle enorme Rohstoffpotentiale, die es zur Verbesserung der nationalen wirtschaftlichen Souveränität und angesichts nationaler Begrenztheit der Rohstoffvorkommen besser zu verwerten gilt. Zusätzlich gefährden die zahlreichen Abfallimporte die nationale Ehre. Die Abfallsituation kann durch bessere Beseitigungs- und Verwertungstechnologien vollständig gemeistert werden. Die bisherigen zivilgesellschaftlichen Widerstände gegen Abfallbehandlungsanlagen haben ihre Ursachen in irrationalen Verdrängungsmechanismen und Mentalitätsrückständen. Demgegenüber ist die Leistungsfähigkeit modernen, wissenschaftlichtechnischen know hows bei der Abfallbeseitigung und -verwertung hervorzuheben. Eine in diesem Sinne moderne Abfallpolitik kann alle Probleme lösen, sofern die gesellschaftlichen Akteure sich ihrer (staatsbürgerlichen) Verantwortung bewusst sind, d.h. Bereitschaft zeigen, die technische Modernisierung zu finanzieren und sich an den entsprechenden Entsorgungs- und Verwertungsinfrastrukturen zu beteiligen. Diese pragmatisch-rationalistische Position gilt es gegen mangelnde staatsbürgerliche Verantwortung der Zivilgesellschaft durchzusetzen. In den Massenmedien hat dieser Diskurs seinen Ort im gesamten konservativen, liberalen oder kritischen Zeitungsspektrum, von ,Le Figaro' über ,Le Monde‘ bis hin zu ,Liberation‘. Träger sind die Regierung, die französische Umweltbehörde ADEME, die Wirtschaft, verschiedene Umweltverbände u. a.m.

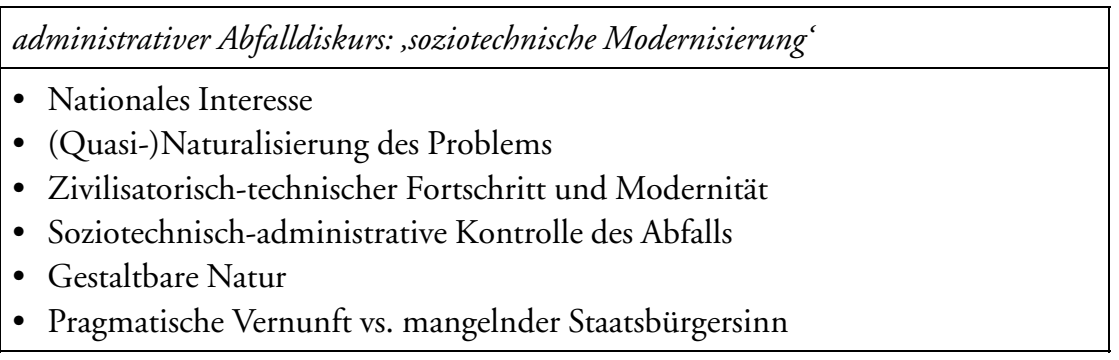

Abbildung 2: Deutungsmuster des hegemonialen Diskurses in der französischen Hausmülldiskussion 
Exemplarische Erläuterung ausgewählter Deutungsmuster

\section{Nationales Interesse}

Der Staat und sein technisch-administrativer Apparat bilden die gemeinwohlorientierte Verkörperung des gesellschaftlichen Gesamtinteresses, dem die einzelnen gesellschaftlichen Akteure sich einsichtig unterordnen (müssen bzw. sollen). Er übernimmt die abfall- und sozio-technische Modernisierung der Zivilgesellschaft durch gezielte wissenschaftlich-(sozio)technische Innovation. Er sichert gleichzeitig die Verwertungsbedingungen der französischen Wirtschaft als Voraussetzung des nationalen Wohlstandes und der nationalen Souveränität, die sich unter anderem am Gradmesser der Autarkie - etwa im Bereich der Energieversorgung und der Verteidigung - bemisst. Der Staat ist Animateur der Zivilgesellschaft, umfassender und unumstrittener Erzieher von Wirtschaft, Kommunen und Staatsbürgern.

\section{Zivilisatorisch-technischer Fortschritt und Modernität}

Damit ist ein Deutungskomplex angesprochen, der das Selbstverständnis der französischen Nation zum Ausdruck bringt. Im linearen Fortschrittsprozess zu mehr Wohlstand und mehr Lebensqualität entfaltet sich zugleich die Rationalität einer aufgeklärten Nation. Die Abfälle bilden hier eine vorübergehende, aber bewältigbare Gefährdung - als ,wilde Deponie' einen Einbruch der Natur in die zivilisatorische Errungenschaft öffentlicher Ordnung und Hygiene, als ,Verschwendungsphänomen' einen Angriff auf moralische und ökonomische Gebote aufgeklärter, effizienter, d. h. vernünftiger Naturnutzung.

Die Rede vom hegemonialen Diskurs deutet schon darauf hin, dass es sich im französischen Fall um eine relative, gleichwohl im Beobachtungszeitraum stabile Machtposition handelt. Dieser Diskurs bzw. seine Protagonisten sprechen unangefochten im Namen des Staates und des nationalen Interesses. Auch wenn dabei einige Gemeinsamkeiten mit dem deutschen strukturkonservativen Abfalldiskurs deutlich werden - etwa hinsichtlich des Vertrauens in die prinzipielle technische Beherrschbarkeit des Abfallaufkommens - kommt es hier jedoch aufgrund der fehlenden öffentlichen Herausforderung nicht zur Legitimationskrise. Wesentliche Impulse seiner Abfallpolitik erhält er denn auch - neben massenmedial vermittelten Appellen an die Wahrnehmung der staatlichen Verantwortung für die nationalen Interessen durch die Antizipation EU-weiter Regulierungsbemühungen in der Abfallpolitik. Damit ist er indirekt von der bundesdeutschen Diskursdynamik betroffen.

Allerdings existiert - bei genauerer Betrachtung - auch in Frankreich ein kulturkritischer Abfalldiskurs. Dieser wird von Netzwerken kritischer Wissenschaftler, auch von Greenpeace (nicht aber von anderen Umweltverbänden), von kleinen Teilen der französischen Grünen und in der Alternativpresse formuliert. Seine Deutungs- 
muster und Positionen sind identisch mit dem deutschen Pendant; er bezieht daher mitunter auch seine Argumentationsgrundlagen. Er ist jedoch in den Massenmedien nicht repräsentiert; er liefert im beobachteten Zeitraum keine legitime Definition der Abfallwirklichkeit. Er erscheint im öffentlichen Resonanzraum Frankreichs weder als um Anerkennung ringende noch als überhaupt existierende Repräsentation der Abfallfrage - ein Paradebeispiel ,kultureller Exklusion': Obwohl es ihn gibt, hat er - bis auf weiteres - keinen Einfluss auf das entsprechende Diskursfeld. ${ }^{15}$ Die legitime, öffentlich anerkannte und sichtbare französische ,Kultur' reproduziert sich im und durch den hegemonialen Diskurs als reiner und gereinigter Fortschrittsrationalismus.

\section{Die permanente Produktion kultureller Unterschiede}

Die vorgestellten Ergebnisse zeigen, wie Kulturen als Diskursfelder, d.h. im Medium der diskursiven Produktion und Reproduktion von symbolischen und institutionellen Strukturen stabilisiert werden und wie sie sich durch spezifische Kombinationen und Auswahlen von Deutungsmustern voneinander unterscheiden, wie sie sich aber auch nach Maßgabe bestimmter ,Sachzwänge' - wie bspw. verfügbare technologische Optionen, transnationale Regulierungsregime - ähneln. Im Falle der Bundesrepublik handelt es sich um eine politische Kultur der Kommunikation über Umweltthemen; für Frankreich kann entsprechend von einer technischen Kommunikationskultur gesprochen werden. Die Attribute ,politisch' und ,technisch` beziehen sich auf die Art und Weise, den Modus oder Stil, wie etwas Thema wird. Eine technische Kultur der Umweltdiskussion orientiert sich an wissenschaftlichtechnischer Expertenrationalität. Diese wird als eindeutig begriffen: klare Aussagenhierarchisierungen sind möglich. Das prägt die Journalisten und ihre Problemwahrnehmung ebenso wie die administrativen Machteliten und Experten, und im $\mathrm{Zu}$ sammenspiel aller die veröffentlichte Interpretation der Müllsituation. Es gibt genau eine richtige Beschreibung des Abfallproblems. Darüber kann nationaler Expertenkonsens als Entscheidungsgrundlage hergestellt werden. Die politische Kultur der Umweltdiskussion erzeugt dagegen eine publikumswirksame Situation der Expertisen- und Interpretationskonkurrenz. Sie eröffnet und ,veröffentlicht' Kontingenzspielräume der Situationsdefinition. Dies gilt auch dann, wenn jeder der konkurrierenden Diskurse für sich selbst genommen davon überzeugt ist, über die über-

15 Ich kann hier nicht näher auf die Potenziale einer entsprechenden Veränderung eingehen. Ein sozialwissenschaftliches Interpretationsangebot zur Transformation diskursiver Regime macht die ,Theorie reflexiver Modernisierung', die katastrophischen Ereignissen und Veränderungen der Risikoakzeptanz einen wichtigen Stellenwert einräumt. Eine diesbezügliche Interpretation des vorliegenden Beispiels findet sich in Keller (1998) und Keller (2000). 
legenen, eindeutigen Argumentationsgrundlagen zu verfügen. Es gibt nicht nur eine staatlich autorisierte Quelle der wissenschaftlich-technischen Wahrheit des Mülls.

Die Erklärung der beobachteten und in diesem Fall nationalstaatlich fixierbaren Unterschiede kann allerdings nicht bei der semantischen Seite von Diskursstrukturen verharren, sondern muss auf die institutionellen Felder und Akteure verweisen, in denen und von denen sie produziert werden: das Zusammenspiel von Ministerien, Experten, Journalisten, Medienbetrieb, Wirtschafts- und Umweltverbänden, die die entsprechenden, ,passenden' Intepretationspartikel formulieren und kommunizieren. Diese institutionellen Gefüge sind selbst geronnene ,Kultur', auf Dauer gestellte, machtvolle Entscheidungstrukturen über legitime Diskussionsbeiträge. Als kontingente, historisch gewachsene und situierte soziokulturelle Gelegenheitsstrukturen formen sie die gesellschaftlichen Definitionsverhältnisse. Was Beobachtern als tiefsitzende nationale Traditionen, Dispositionen und Sensibilitäten für Umweltprobleme erscheint, ist in genau diesem Sinne Ergebnis der beständigen aktiven Herstellungsleistung kollektiver Akteure im Rahmen eines bestehenden institutionell-kulturellen Gefüges. Die verschiedenen, in Diskurse verstrickten Akteure erzeugen im Rückgriff auf tradierte und resonanzfähige Interpretationsmuster unterschiedliche öffentliche (massenmediale) Repräsentationen von Umwelt. Sie verankern spezifisch gefilterte Deutungsmuster von Umweltproblemen im ,kollektiven Gedächtnis‘. Die Strukturierungen der Diskurse sind die kulturelle Tradition, die in Institutionen geronnen ist und im Abfalldiskurs symbolisch legitimiert und fortgeschrieben wird, d. h. sich im Medium der Abfalldebatte reproduziert.

Die materialen Abfallpolitiken beider Länder unterscheiden sich, wie weiter oben erwähnt, trotz der unterschiedlichen Diskussionsverläufe nicht prinzipiell, sondern graduell. Dafür gibt es drei Gründe: Zum einen bestehen viele Gemeinsamkeiten der Probleminterpretation zwischen dem deutschen strukturkonservativen und dem französischen technisch-administrativen Diskurs, die jeweils über die materiale $\mathrm{Ab}$ fallpolitik im Untersuchungszeitraum entscheiden. Dazu zählt etwa der jeweilige argumentative Bezug auf ,Nachhaltigkeit' im Rahmen der abfallpolitischen Regulierungen Anfang der 1990er Jahre, in der Zeit der ersten Rio-Konferenz. Zum zweiten resultiert der ,Vorsprung' der bundesdeutschen Abfallpolitik aus der hohen öffentlichen Legitimität des herausfordernden kulturkritischen Abfalldiskurs, die den regierungstragenden strukturkonservativen Abfalldiskurs zu immer weiterreichenden Maßnahmen zwingt. Daraus entsteht die Innovationsdynamik der bundesdeutschen Abfalldiskussion. Frankreichs Abfallpolitik der frühen 1990er Jahre ist dann drittens als Import der bundesdeutschen Diskursdynamik durch internationale Regulierungsvernetzung - vermittelt über die EU - zu verstehen. Bei dem erwähnten letzten Punkt handelt es sich - bezogen auf die deutschen Diskurse - um eine Art ,externen Diskurseffekt'. Um einen solchen Zusammenhang zu erkennen, muss sich die Diskursforschung allerdings von einer allzu starken Textkonzentration 
lösen und Wissen über Kontextprozesse - in diesem Fall rechtliche Regulierungsziele auf EU-Ebene - einbeziehen. ,Diskursextern' sind diese freilich nur in Bezug auf die hier untersuchten öffentlichen, massenmedialen Diskurse. Je tiefer die Diskursanalyse bspw. in die internen institutionellen Diskurse der französischen Politik und Administration eintaucht, desto stärker kann sie - wie die im Rahmen der durchgeführten Untersuchung zu Informationszwecken ausgewerteten Interviews und administrationsinternen abfallpolitischen Dokumente zeigen - die hier genannten Zusammenhänge als diskursiv manifestierte rekonstruieren. Allerdings muss jede sozialwissenschaftliche Diskursforschung, die sich von der reinen Textebene löst will sie sich nicht in der Unendlichkeit der Rekonstruktion verlieren - bestimmte Kontext-Parameter ihres Untersuchungsgegenstandes als gegeben voraussetzen, wohl wissend, dass auch solche (bspw. institutionellen) Kontexte als kristallisierte Diskurse begriffen werden können.

\section{Literatur}

Berger, Peter/Luckmann, Thomas (1969): Die gesellschaftliche Konstruktion der Wirklichkeit. Frankfurt a.M.: Fischer.

Boltanski, Luc/Chiapello, Eve (1999): Le nouvel esprit du capitalisme. Paris: Gallimard.

Bourdieu, Pierre (1992): Die verborgenen Mechanismen der Macht. Hamburg: VSA.

Bourdieu, Pierre (1993): Sozialer Sinn. Frankfurt a.M.: Suhrkamp.

Burgess, Jacquie (1990): The production and consumption of environmental meanings in the mass media: a research agenda for the 1990s. In: Transactions of the Institute of British Geographers 15. 139-161

Clifford, James (1992): Travelling Cultures. In: Grossberg, Lawrence/Nelson, Cary/ Treichler, Paula (Hg.): Cultural Studies. New York. 96-116

Foucault, Michel (1974a): Die Ordnung der Dinge. Frankfurt a.M.: Suhrkamp [1966].

Foucault, Michel (1974b): Die Ordnung des Diskurses. München: Hanser.

Foucault, Michel (1981): Archäologie des Wissens. Frankfurt a.M.: Suhrkamp [1969].

Gamson, William A./Modigliani, Andre (1989): Media discourse and public opinion on nuclear power. A constructionist approach. In: American Journal of Sociology, $95.1-73$

Geertz, Clifford (1973): The Interpretation of Cultures. New York: Basic Books.

Giddens, Anthony (1988): Die Konstitution der Gesellschaft. Gründzüge einer Theorie der Strukturierung. Frankfurt a.M.: Campus. 
Gusfield, John R. (1981): The Culture of Public Problems: Drinking-Driving and the Symbolic Order. Chicago: University Press.

Hitzler, Ronald/Honer, Anne (1994): Qualitative Methoden. In: Kriz, Jürgen/Nohlen, Dieter/Schultze Rainer-Olaf(Hg.): Lexikon der Politik, Bd. 2: Politikwissenschaftliche Methoden. München: Beck. 389-395.

Hitzler, Ronald/Honer, Anne (Hg.) (1997): Sozialwissenschaftliche Hermeneutik. Opladen: Leske und Budrich.

Keller, Reiner (1997a): Die Umweltberichterstattung im Spiegel der Medienforschung. In: Brand, Karl-Werner/Eder, Klaus/Poferl, Angelika (Hg.): Ökologische Kommunikation in Deutschland. Opladen: Westdeutscher Verlag. 62-72.

Keller, Reiner (1997b): Diskursanalyse. In: Hitzler, Ronald/Honer, Anne (Hg.): Sozialwissenschaftliche Hermeneutik. Opladen: Leske und Budrich. 309-334.

Keller, Reiner (1998): Müll - Die gesellschaftliche Konstruktion des Wertvollen. Opladen: Westdeutscher Verlag.

Keller, Reiner (2000): Der Müll in der Öffentlichkeit. Reflexive Modernisierung als kulturelle Transformation. In: Soziale Welt 51. 245-266.

Keller, Reiner (2001): Wissenssoziologische Diskursanalyse. In: Keller, Reiner/Hirseland, Andreas/Schneider, Werner/Viehöver, Willy (Hg.): Handbuch Sozialwissenschaftliche Diskursanalyse Bd. 1: Theorien und Methoden. Opladen: Leske und Budrich. 113-144.

Keller, Reiner (2003a): Diskursforschung. Eine Einführung für SozialwissenschaftlerInnen. Opladen: Leske und Budrich.

Keller, Reiner (2003b): Der Müll der Gesellschaft. Beispiel einer Wissenssoziologischen Diskursanalyse. In: Keller, Reiner/Hirseland, Andreas/Schneider, Werner/ Viehöver, Willy (Hg.): Handbuch Sozialwissenschaftliche Diskursanalyse. Bd. 2: Forschungspraxis. Opladen: Leske und Budrich.

Keller, Reiner/Hirseland, Andreas/Schneider, Werner/Viehöver, Willy (Hg.) (2001): Handbuch Sozialwissenschaftliche Diskursanalyse Bd. 1: Theorien und Methoden. Opladen: Leske und Budrich.

Keller, Reiner/Hirseland, Andreas/Schneider, Werner/Viehöver, Willy (Hg.) (2003): Handbuch Sozialwissenschaftliche Diskursanalyse. Bd. 2: Forschungspraxis. Opladen: Leske und Budrich.

Lüders, Christian/Meuser, Michael (1997): Deutungsmusteranalyse. In: Hitzler, Ronald./Honer, Anne (Hg.): Sozialwissenschaftliche Hermeneutik. Opladen: Leske und Budrich, 57-80.

Plaß, Christine/Schetsche, Michale (2001): Grundzüge einer wissenssoziologischen Theorie sozialer Deutungsmuster. In: Sozialer Sinn 1, 511-536.

Poferl, Angelika (1997): Der strukturkonservative Risikodiskurs. Eine Analyse der Tschernobyl ,Media Story' in der Frankfurter Allgemeinen Zeitung. In: Brand, 
Karl-Werner/Eder, Klaus/Poferl, Angelika (Hg.): Ökologische Kommunikation in Deutschland. Opladen: Westdeutscher Verlag. 106-154.

Potter, Jonathan/Wetherell, Margaret (1995): Soziale Repräsentationen, Diskursanalyse und Rassismus. In: Flick, Uwe (Hg.): Psychologie des Sozialen. Repräsentationen in Wissen und Sprache. Reinbeck: Rowohlt. 177-199.

Reckwitz, Andreas (2000): Die Transformation der Kulturtheorien. Weilerswist: Vellbrück.

Sahlins, Marshall (1981): Kultur und praktische Vernunft. Frankfurt a.M.: Suhrkamp.

Schiffauer, Werner (1995): Sie verlassen die geschützte Zone! taz, 14.11.95. 14-15.

Soeffner, Hans-Georg/Hitzler, Ronald (1994): Hermeneutik als Haltung und Handlung. In: Schröer, N. (Hg.): Interpretative Sozialforschung. Opladen: Westdeutscher Verlag. 28-55.

Swidler, Anne (1986): Culture in action: Symbols and Strategies. In: American Sociological Review Nr. 51/1986. 273-286.

Viehöver, Willy (2001): Diskurse als Narrationen. In: Keller, Reiner/Hirseland, Andreas/Schneider, Werner/Viehöver, Willy (Hg.): Handbuch Sozialwissenschaftliche Diskursanalyse Bd. 1: Theorien und Methoden. Opladen: Leske und Budrich. 177-206.

Viehöver, Willy (2003): Die Wiederverzauberung des sublunearen Raumes. Beispiel der narrativen Diskursanalyse. In: Keller, Reiner/Hirseland, Andreas/Schneider, Werner/Viehöver, Willy. (Hg.): Handbuch Sozialwissenschaftliche Diskursanalyse Bd. 2: Forschungspraxis. Opladen: Leske und Budrich. 


\section{Die Gegenwart der Vergangenheit}


